\title{
A systemic study of lipid metabolism regulation in salmon fingerlings and early juveniles fed plant oil
}

\author{
Yang Jin ${ }^{1 *}$, Rolf E. Olsen ${ }^{1}$, Gareth B. Gillard ${ }^{2}$, Mari-Ann Østensen ${ }^{1}$, Sven A. Korsvoll ${ }^{3}$, Nina Santi ${ }^{3}$, \\ Jon O. Vik ${ }^{4}$, Simen R. Sandve ${ }^{4}$ and Yngvar Olsen ${ }^{1}$ \\ ${ }^{1}$ Department of Biology, Norwegian University of Science and Technology, N-7491 Trondheim, Norway \\ ${ }^{2}$ Department of Chemistry, Biotechnology and Food Science, Norwegian University of Life Sciences, N-1430 Ås, Norway \\ ${ }^{3}$ AquaGen AS, Postboks 1240, Sluppen, N-7462 Trondheim, Norway \\ ${ }^{4}$ Department of Animal and Aquacultural Sciences, Centre for Integrative Genetics, Norwegian University of Life Sciences, \\ $N-1430$ As, Norway \\ (Submitted 5 March 2018 - Final revision received 10 June 2018 - Accepted 13 June 2018 - First published online 1 August 2018)
}

\section{Abstract}

In salmon farming, the scarcity of fish oil has driven a shift towards the use of plant-based oil from vegetable or seed, leading to fish feed low in long-chain PUFA (LC-PUFA) and cholesterol. Atlantic salmon has the capacity to synthesise both LC-PUFA and cholesterol, but little is known about the regulation of synthesis and how it varies throughout salmon life span. Here, we present a systemic view of lipid metabolism pathways based on lipid analyses and transcriptomic data from salmon fed contrasting diets of plant or fish oil from first feeding. We analysed four tissues (stomach, pyloric caeca, hindgut and liver) at three life stages (initial feeding $0.16 \mathrm{~g}, 2.5 \mathrm{~g}$ fingerlings and $10 \mathrm{~g}$ juveniles). The strongest response to diets higher in plant oil was seen in pyloric caeca of fingerlings, with up-regulation of thirty genes in pathways for cholesterol uptake, transport and biosynthesis. In juveniles, only eleven genes showed differential expression in pyloric caeca. This indicates a higher requirement of dietary cholesterol in fingerlings, which could result in a more sensitive response to plant oil. The LC-PUFA elongation and desaturation pathway was down-regulated in pyloric caeca, probably regulated by srebp1 genes. In liver, cholesterol metabolism and elongation and desaturation genes were both higher on plant oil. Stomach and hindgut were not notably affected by dietary treatment. Plant oil also had a higher impact on fatty acid composition of fingerlings compared with juveniles, suggesting that fingerlings have less metabolic regulatory control when primed with plant oil diet compared with juveniles.

\section{Key words: Atlantic salmon: Fingerlings: Juvenile fish: Transcriptomics: Plant oil: Cholesterol}

Atlantic salmon (Salmo salar) is one of the most economically important aquaculture species constituting $35 \%$ of global marine fish production ${ }^{(1)}$. The production of farmed salmon increased rapidly from 500 metric tons in 1970 to more than $2 \cdot 3$ million metric tons in $2014^{(1)}$. However, shortages of sufficient fishmeal and oils have led to massive substitutions (60\%) by plant ingredients in salmon diets ${ }^{(2)}$. This includes an increase in plant oils (PO) from 0\% in the diet in 1990 to $19.2 \%$ in $2013^{(2)}$. As the lipid composition of PO is significantly different from that in fish oil (FO), this can influence growth and performance of salmon at early developmental stages.

The gastrointestinal tract of salmon consists of three major regions, the stomach, pyloric caeca and midgut, and hindgut, each of them with separate functions in lipid metabolism. Most studies on dietary lipid utilisation have focused on the pyloric caeca or midgut as they are the predominant regions for lipid absorption and transport in salmon ${ }^{(3-6)}$. The hindgut could also be involved in absorption and transport of lipids, as both histological and genetical studies have suggested an existence of lipoproteins in the region ${ }^{(7,8)}$. The stomach is primarily responsible for mechanical and chemical digestion of feed, but may also be involved in absorption of SCFA, Na and water ${ }^{(9)}$. Similar to other teleosts, the stomach and pyloric caeca of salmon are the last organs to be completed during ontogenetic development of the digestive system ${ }^{(10,11)}$. The stomach and hindgut are distinguishable $7 \mathrm{~d}$ after hatching, whereas the pyloric caeca occurs $20 \mathrm{~d}_{\text {later }}{ }^{(11)}$. Although the whole intestine of salmon is prepared for digestion and absorption of external feed before the yolk sac is depleted $^{(11)}$, it is still unclear whether the capacities of digestion and absorption in salmon fingerlings are developed enough to tolerate a plant-based diet with high PO contents. The adult salmon can tolerate partial replacement of PO in diet without

Abbreviations: ARA, arachidonic acid; DEG, differential expressed genes; elovl, elongase; fads, fatty acyl desaturase; FO, fish oil; LC-PUFA, long-chain PUFA; PO, plant oil; PtdCho, phosphatidylcholine; PtdEtn, phosphatidylethanolamine.

* Corresponding author: Y. Jin, fax +47 73596311, email jinyangye119@hotmail.com 
influencing growth ${ }^{(2,12-16)}$. However, feeding PO could still cause massive accumulation of lipid droplets in enterocytes of the pyloric caeca, indicating abnormal lipid transport across the intestinal region ${ }^{(4,5,17)}$. The liver is also important for synthesising and transporting lipids in salmon. Previous studies mostly used liver tissue to evaluate PO-induced changes of lipid metabolism in large salmon ${ }^{(16,18)}$

Like all vertebrates, salmon require cholesterol for growth and development as it is involved in many biological functions, such as formation of cell membranes and provision of bile acids $^{(19)}$. Cholesterol homoeostasis in the fish body is achieved by balancing dietary intake, de novo synthesis in the body and excretion as bile acids. PO is devoid of cholesterol, but contains several types of phytosterols ${ }^{(20,21)}$. Although these phytosterols have lower bioavailability, they can further reduce the absorption of available dietary cholesterol ${ }^{(20)}$. This would increase the demand on de novo synthesis, as shown by the up-regulation of cholesterol biosynthesis pathways in liver ${ }^{(18,22)}$. Whereas adult salmon are self-sufficient in cholesterol when on a PO diet ${ }^{(23)}$, this ability in salmon fingerlings is largely unknown.

Atlantic salmon also require dietary supplement of long-chain PUFA (LC-PUFA, $\geq \mathrm{C}_{20}$ ) such as DHA (22:6n-3), EPA (20:5n-3) and arachidonic acid (ARA, 20:4n-6) for optimum growth and development ${ }^{(24)}$. The requirement of LC-PUFA for salmon as for other fish is generally higher for fingerlings than juvenile or adult stages ${ }^{(25-27)}$. However, as PO does not contain LC-PUFA, their content will be reduced upon feeding PO diets. Salmonids are capable of elongating and desaturating 18:3n-3 and $18: 2 n-6$ to LC-PUFA ${ }^{(28)}$, and fatty acyl desaturase (fads) and elongase $(e l o v l)$ genes are often up-regulated in salmon upon PO feeding ${ }^{(15)}$. However, the impact of elongation and desaturation on LC-PUFA level in salmon is often much less than that of diet ${ }^{(29)}$. Furthermore, the capacity to elongate and desaturate is largely unknown in salmon fingerlings and early juveniles compared with larger individuals.

All salmonids have experienced an extra round of wholegenome duplication compared with other fish species. This salmonid-specific duplication (Ss4R) occurred in a common ancestor of all salmonids approximately 100-80 million years ago $^{(30,31)}$. To date, approximately $55 \%$ of the Ss $4 \mathrm{R}$ gene duplicates are still retained as expressed genes in Atlantic salmon ${ }^{(32)}$. Some of the Ss4R duplicates have been found to have distinct regulation in different tissues ${ }^{(8)}$, developmental stages $^{(8)}$ and dietary treatment ${ }^{(22)}$. This has markedly increased the complexity of understanding lipid metabolism pathways in salmon, meaning the functional divergence of gene duplicates must be taken into consideration in diet-induced gene expression studies.

In the present study, we applied transcriptomic and lipid analysis on the stomach, pyloric caeca, hindgut and liver of salmon fed either PO- or FO-enriched diets. The developmental aspect included first feeding $(0.16 \mathrm{~g})$, fingerling $(2.5 \mathrm{~g})$ and juvenile $(10 \mathrm{~g})$ salmon. The overarching goal was to achieve a systemic overview of PO-induced lipid metabolism changes in salmon fingerlings and juveniles. Expressional differences between Ss4R duplicate genes were also taken into consideration for studying the salmonid-specific features of genetic regulation of lipid metabolism.

\section{Methods}

\section{Fish, diets and experimental plan}

This study was carried out within the Norwegian animal welfare act guidelines, in accordance with EU regulation (EC Directive 86/609/EEC). Atlantic salmon eggs were hatched and cultivated at AquaGen Breeding Centre. The fish were fed either PO or FO diets (Cargill Innovation Centre) from first feeding up to $100 \mathrm{~d}$. The PO used in this study was a mixture of palm and linseed oil. The composition of the two diets and their fatty acids are summarised in Table 1. A previous experiment used the same diets for studying the effect of $\mathrm{PO}$ on gene regulation in larger salmon before and after smoltification ${ }^{(22)}$. We sampled three fish from each dietary group at days $0(0.16 \mathrm{~g}$, before first feeding), 65 (2.5 g, fingerlings) and 100 (10 g, juvenile) after first feeding. The fish were euthanised by $1 \mathrm{~g} / \mathrm{l}$ MS-222 (Finquel, Argent Chemical Laboratories, Inc.) buffered with the same amount of sodium bicarbonate before dissection in a sterile Petri dish filled with RNAlater. Samples of stomach, pyloric caeca, hindgut and liver were dissected, cleaned of connective tissues and intestinal contents and immediately transferred to $1 \mathrm{ml}$ of RNAlater for RNAseq analysis. Tissues were stored for $24 \mathrm{~h}$ at $4^{\circ} \mathrm{C}$ for sufficient penetration of RNAlater before being transferred to $-80^{\circ} \mathrm{C}$ for storage. Tissues of stomach, pyloric caeca and hindgut for lipid analysis were flash-frozen in liquid nitrogen and stored at $-80^{\circ} \mathrm{C}$.

\section{Lipid class and fatty acid analyses}

Total lipids were extracted from salmon tissues using the method of Folch et al. ${ }^{(33)}$. Lipid classes were separated using the double development high-performance TLC method of Olsen \& Henderson ${ }^{(34)}$. Extracted total lipid was applied onto $10 \times 10 \mathrm{~cm}$ silica plates (Merck) for lipid class separation. The plates were developed using methyl acetate-isopropanolchloroform-methanol-0.25\% $\mathrm{KCl}(25: 25: 25: 10: 9$, by vol.) to separate polar lipids and hexane-diethyl ether-glacial acetic acid (80:20:2, by vol) for separation of neutral lipids. The phosphatidylcholine (PtdCho), phosphatidylethanolamine (PtdEtn) and TAG fractions were detected under UV light, scraped off and directly methylated with sulphuric acidmethanol $(2: 98, \mathrm{v} / \mathrm{v})$ in isooctane at $50^{\circ} \mathrm{C}$ overnight. Isooctane and $5 \% \mathrm{NaCl}$ were then added to the mixture and centrifuged at $1640 \mathrm{~g}$ for $3 \mathrm{~min}$ at $4^{\circ} \mathrm{C}$. The fatty acid methyl esters in the upper phase were analysed on an Agilent 7890B gas chromatograph with flame ionisation detector (Agilent Technologies). Fatty acid data were generated from two individual samples with two technical replicates $(2 \times 2)$.

\section{RNA extraction and transcriptomic analysis}

Three individual samples per tissue and experimental condition were used as biological replicates. Total RNA was extracted using RNeasy Plus Universal Kits (Qiagen). RNA concentration and purity were assessed by Nanodrop 8000 (Thermo Scientific) and RNA integrity was checked by Agilent 2100 Bioanalyzer (Agilent Technologies). Libraries were prepared by using TruSeq Stranded mRNA Library Prep Kit (Illumina), according to the manufacturer's instructions. Samples were sequenced using 100-bp single-end high-throughput mRNA 
sequencing (RNA-Seq) on Illumina Hiseq 2500 (Illumina) in Norwegian Sequencing Centre. The raw sequences are publicly available on European Nucleotide Archive under accession number PRJEB21981. The raw sequence data for FO samples were used in a previous study to understand the transcriptional development of phospholipid and lipoprotein metabolism in salmon fry ${ }^{(8)}$.

\section{Statistical analysis and data mining}

Fatty acid analysis data were checked for statistical significance by using one-way ANOVA with Tukey's multiple comparison test, and differences were considered significant when $P<0.05$.

For transcriptomic analysis, the quality control and counting of the read sequences were performed by the same method as described previously ${ }^{(22)}$. The uniquely mapped reads, aligned to exon regions, were counted for each gene in the salmon genome annotation (ICSASG_v2). Genes were filtered by a minimum count level of at least 1 count per million (CPM) in two or more samples, to remove genes with too few counts. For each tissue type (stomach, pyloric caeca, hindgut and liver), at each developmental stage ( $2.5 \mathrm{~g}$ fingerlings and $10 \mathrm{~g}$ juvenile), a differential expression analysis (DEA) was performed comparing PO with FO samples. For visually comparing expression levels between different genes and tissues, normalised counts in the form of transcripts per million values were generated. Raw gene counts were first divided by their mRNA length in $\mathrm{kb}$ to normalise for transcript length, and then divided by the total number of counts from each library to normalise for sequencing depth. For comparison of expression between Ss4R duplicated genes, an Ss4R duplicate gene list defined in a previous study was applied $^{(22)}$. The present study had a special focus on genes involved in lipid metabolism pathways; therefore, 353 salmon lipid genes were selected from previous orthologue annotations to Kyoto Encyclopedia of Genes and Genomes (KEGG) database terms ${ }^{(22)}$. The list of lipid genes with NCBI identifiers is shown in online Supplementary Table S1. RNA-Seq analysis was performed using $\mathrm{R}$ (version 3.4.1). The KEGG ontology enrichment analysis (KOEA) and DEA were conducted using $\mathrm{R}$ package edgeR. DEA was conducted using pairwise exact tests to produce gene fold changes and $P$ values. Genes with a false discovery rate-adjusted $P$ value $(q)<0.05$ were considered differentially expressed between two test conditions. Hypergeometric test was applied in KOEA, based on the number of differential expressed genes (DEG) $v$. total genes annotated to each KEGG ontology (KO) term, and differences were considered significant when $P<0 \cdot 05$. A heatmap was drawn using $\mathrm{R}$ with the pheatmap package. The pathway diagram was produced using PathVisio version 3.2.4. All other figures were produced using $\mathrm{R}$ package ggplot2.

\section{Results}

\section{Intestinal fatty acid composition}

The percentage of major fatty acids in PtdCho, PtdEtn and TAG of salmon after feeding PO compared with FO is shown in Table 2. Other fatty acids are shown in online Supplementary Table S2. Regardless of tissue, fatty acid composition of
TAG was similar to the fed diet (Table 2). The content of $18: 1 n-9,18: 2 n-6$ and $18: 3 n-3$ increased after feeding PO compared with FO, whereas the content of $20: 1 n-9,22: 1 n-11$, EPA and DHA decreased. The number of significantly $(P<0.05)$ changed fatty acids was much higher in fingerlings $(2.5 \mathrm{~g})$ than in juveniles $(10 \mathrm{~g}$ ), and the changes in percentage of fatty acids were also higher. For example, the percentage of DHA in TAG was reduced by over $50 \%$ in PO compared with FO-fed fingerlings, whereas in juveniles the TAG-DHA was reduced by $30 \%$.

The dominant fatty acids of PtdCho were 16:0, 18:1n-9, EPA and DHA, and feeding of PO had a different impact on the PtdCho of different intestinal regions and developmental stages of salmon (Table 2). The amount of 16:0 was relatively stable regardless of diet, except for a slight increase in stomach and hindgut of juvenile salmon fed PO. The content of $18: 1 n-9$ increased in all three tissues of fingerlings after feeding PO, but in juveniles the effects were less pronounced in pyloric caeca, with no effects observed on stomach and hindgut. The percentage of $18: 2 n-6$ and $18: 3 n-3$ increased in both fingerling and juvenile salmon fed PO compared with FO, which was in line with the high amount of the two fatty acids in the PO diet (Tables 1 and 2). Reductions of ARA and EPA were only observed in stomach and pyloric caeca of fingerlings after feeding PO, whereas DHA decreased in all three tissues. The EPA and DHA levels were not affected in pyloric caeca of juvenile salmon when fed PO diet.

The composition of fatty acids in PtdEtn was less influenced by $\mathrm{PO}$ diet compared with the fatty acids in other lipid classes (Table 2). The content of 16:0 was not changed when fed PO, whereas a slight increase in 18:0 was observed. The percentage

Table 1. Composition of plant oil (PO) and fish oil (FO) diets

\begin{tabular}{lcc}
\hline & FO diet & PO diet \\
\hline Diet composition & & \\
Fishmeal & 40 & 40 \\
Plant meals & $41 \cdot 5$ & $41 \cdot 5$ \\
Additives & $3 \cdot 5$ & $3 \cdot 5$ \\
North Atlantic fish oil & $15 \cdot 1$ & 0 \\
Linseed oil & 0 & $9 \cdot 7$ \\
Palm oil & 0 & $5 \cdot 4$ \\
Protein (\%) & 51 & 51 \\
Fat (\%) & 21 & 21 \\
Fatty acid composition (\%) & & \\
$14: 0$ & $6 \cdot 9$ & $1 \cdot 8$ \\
$16: 0$ & $13 \cdot 9$ & $17 \cdot 9$ \\
$18: 0$ & $1 \cdot 8$ & $3 \cdot 2$ \\
Total saturates & $23 \cdot 1$ & $23 \cdot 2$ \\
$16: 1 n-7$ & $4 \cdot 6$ & $1 \cdot 1$ \\
$18: 1 n-9$ & $11 \cdot 3$ & $21 \cdot 0$ \\
$20: 1 n-9$ & $10 \cdot 3$ & $1 \cdot 8$ \\
$22: 1 n-11$ & $14 \cdot 5$ & $3 \cdot 0$ \\
Total monoenes & $40 \cdot 7$ & $26 \cdot 9$ \\
$18: 2 n-6$ & $6 \cdot 7$ & $12 \cdot 9$ \\
$20: 4 n-6$ & $0 \cdot 2$ & $0 \cdot 1$ \\
Total $n-6$ PUFA & $7 \cdot 0$ & $13 \cdot 1$ \\
$18: 3 n-3$ & $3 \cdot 5$ & $29 \cdot 3$ \\
$20: 3 n-3$ & $0 \cdot 2$ & $0 \cdot 1$ \\
$20: 5 n-3$ (EPA) & $7 \cdot 0$ & $1 \cdot 7$ \\
$22: 5 n-3$ & $0 \cdot 6$ & $0 \cdot 2$ \\
$22: 6 n-3$ (DHA) & $7 \cdot 7$ & $2 \cdot 5$ \\
Total $n-3$ PUFA & $19 \cdot 0$ & $33 \cdot 8$ \\
\hline & & \\
\hline
\end{tabular}


Y. Jin et al.

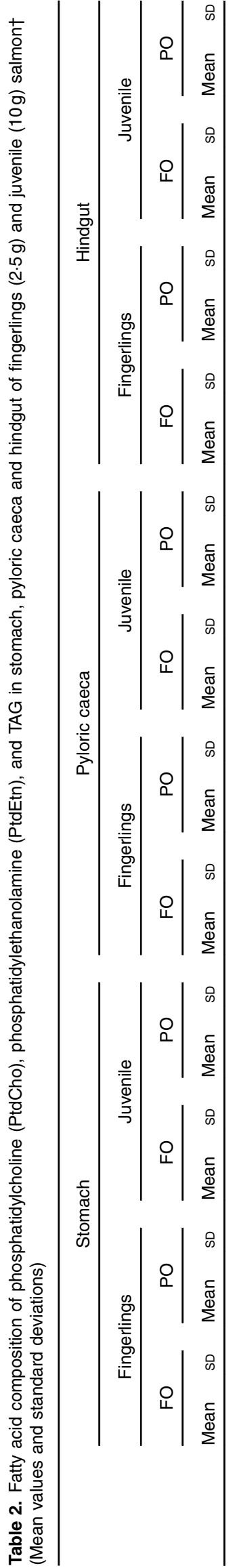

nethota

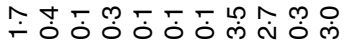

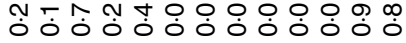

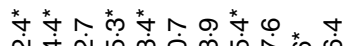

ด

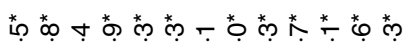

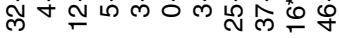

맘용

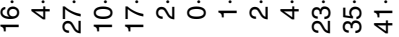

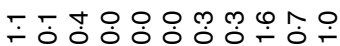

L

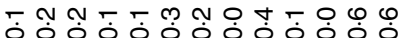

N $+\infty \infty$ のर

OOANG- OHNN

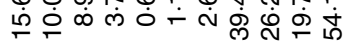

มิ山

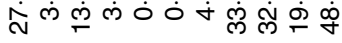

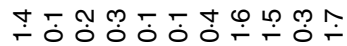

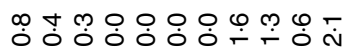

둔

ก

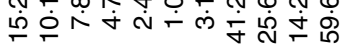

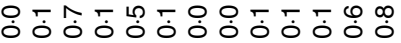

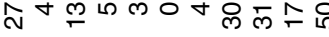

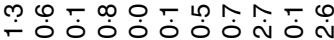



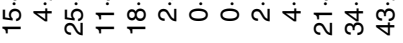

$0 N N O N$ N

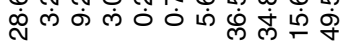

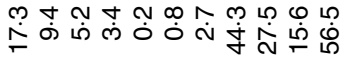

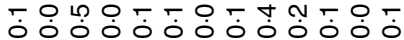

L N N

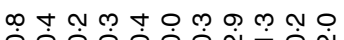

œ -

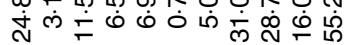

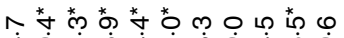

ப்

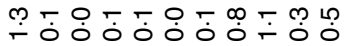

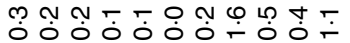

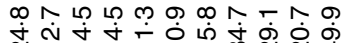

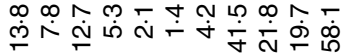

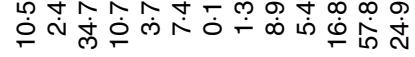

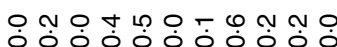

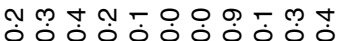

-

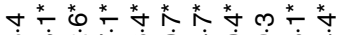

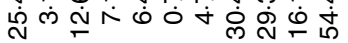

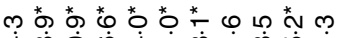

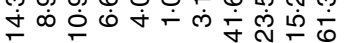

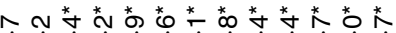

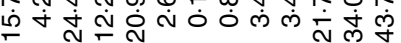

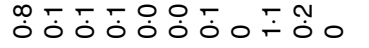

N

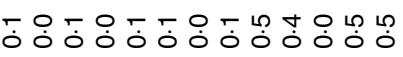

私

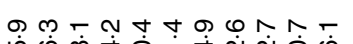

0 d ம்

ex m+d m

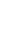

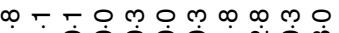

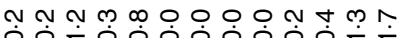

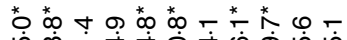

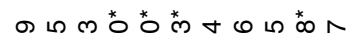

ف웜

颌 ம்

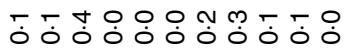

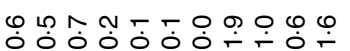

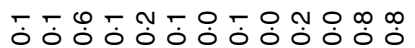

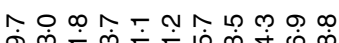

बं लं

то一兀

$\forall$ 皮

ஸे

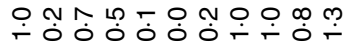

மூல்

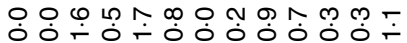

ナ

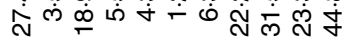

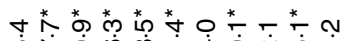

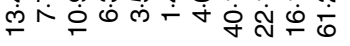

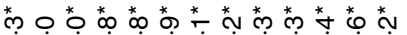
ம்

mó

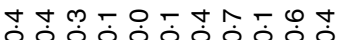

ז்́ 过

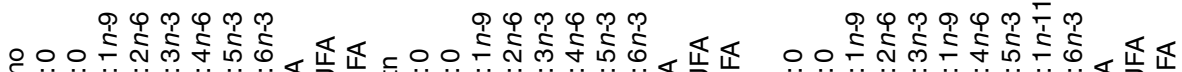

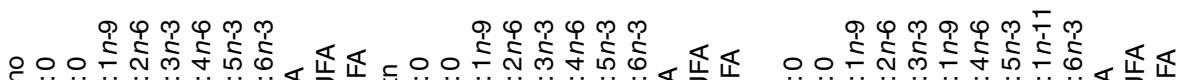
家

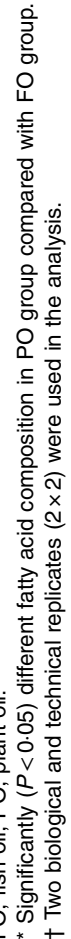


of $18: 1 n-9$ was only increased in fingerlings, similarly observed in PtdCho. Increases in $18: 2 n-6$ and $18: 3 n-3$ were also found when feeding PO compared with FO. The content of EPA was significantly $(P<0.05)$ reduced in pyloric caeca of salmon fingerlings; otherwise, no difference was observed between PO and FO groups. Over $40 \%$ of DHA were found in PtdEtn of all intestinal regions, and the percentage was only reduced in stomach of fingerlings when fed PO.

\section{Overview of intestinal transcriptome and KEGG ontology analysis}

An average of twenty-two million reads were collected from each library, of which approximately $85 \%$ were mapped to the salmon genome. From a total of 81597 genes currently annotated, 35381 genes met a minimum level of expression for DEA (at least $1 \mathrm{CPM}$ in two or more samples). The highest number of DEG between PO and FO treatments was 2214 found in pyloric caeca of fingerlings, which reduced to 486 DEG in juveniles. The number of DEG in stomach also decreased from 437 in fingerlings to six in juveniles. On the other hand, the DEG in the hindgut and liver of fingerlings were 142 and 40, respectively, which both increased to approximately 500 DEG in juveniles.

The highest number of significantly $(P<0.05)$ regulated KEGG pathways was found in pyloric caeca of fingerlings (Fig. 1).

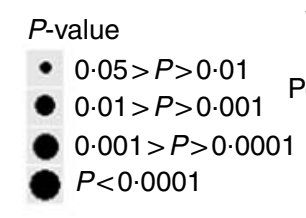

Tissue
$\begin{aligned} & \text { stomach } \\ & \text { pyloric caeca } \\ & \text { hindgut } \\ & \text { liver }\end{aligned}$
These included ten pathways involved in lipid metabolism, five pathways in carbohydrate metabolism and six pathways in protein metabolism. Fewer KEGG pathways were significantly $(P<0.05)$ regulated in pyloric caeca of juvenile salmon, including only three pathways involved in lipid metabolism. The pathway of steroid biosynthesis was highly regulated both in fingerling and juvenile salmon fed PO compared with those fed FO. Feeding of PO also caused significant $(P<0.05)$ changes of three pathways of fatty acid metabolism in pyloric caeca of fingerlings, indicating a group of DEG involved in fatty acid metabolism. Pathways of glycerolipid and glycerophospholipid metabolism were also changed in pyloric caeca of fingerlings fed PO compared with FO. Many pathways were changed in stomach of fingerlings fed PO diet, whereas few pathways were changed in the same tissue of juvenile salmon. In the hindgut, three pathways of lipid metabolism were changed in fingerlings after feeding PO, whereas no lipid metabolism pathway was changed in juveniles. However, thirteen pathways in carbohydrate and protein metabolism were significantly regulated in juvenile salmon fed PO. No lipid metabolism pathway was regulated in liver when feeding PO diet. All other significantly $(P<0.05)$ regulated pathways in stomach, pyloric caeca, hindgut and liver are shown in online Supplementary Table S3.
Vascular smooth muscle contraction Ca signalling pathway Porphyrin and chlorophyll metabolism

Terpenoid backbone biosynthesis $\mathrm{ABC}$ transporters $\alpha$-Linolenic acid metabolism Linoleic acid metabolism Arachidonic acid metabolism Steroid hormone biosynthesis Primary bile acid biosynthesis Glycerophospholipid metabolism Steroid biosynthesis Glycerolipid metabolism Fatty acid biosynthesis

Pentose phosphate pathway Glyoxylate and dicarboxylate metabolism Fructose and mannose metabolism Pyruvate metabolism Amino sugar and nucleotide sugar metabolism Ascorbate and aldarate metabolism Pentose and glucuronate interconversions Glycolysis / Gluconeogenesis

Glycine, serine and threonine metabolism Phenylalanine, tyrosine and tryptophan biosynthesis Tryptophan metabolism Phenylalanine metabolism Tyrosine metabolism Histidine metabolism Arginine and proline metabolism Valine, leucine and isoleucine degradation Alanine, aspartate and glutamate metabolism Arginine biosynthesis
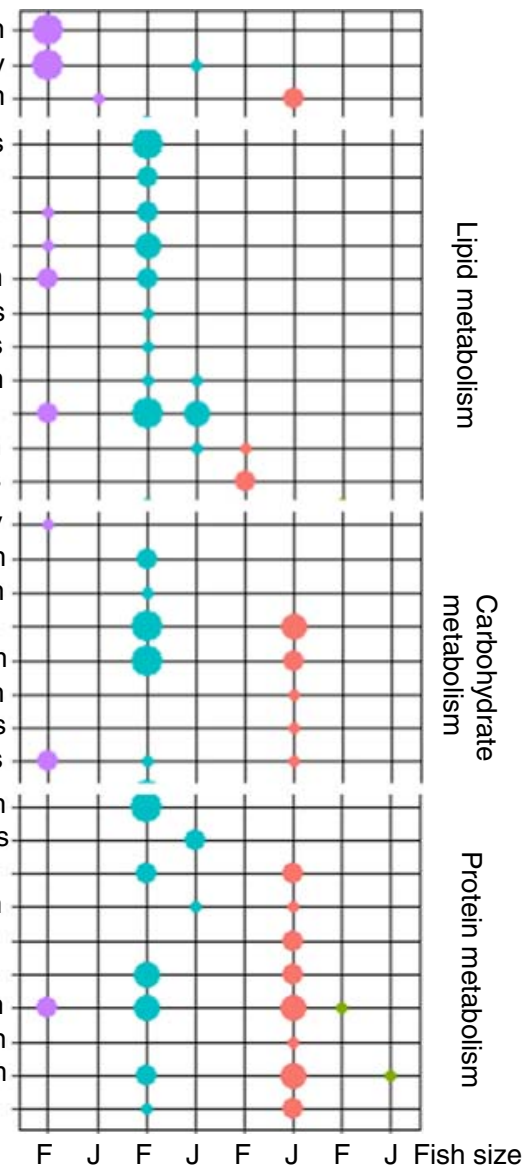

Fig. 1. Bubble graph of KEGG ontology enrichment analysis results for stomach, pyloric caeca, hindgut and liver of fingerling ( $F, 2.5 \mathrm{~g})$ and juvenile $(\mathrm{J}, 10 \mathrm{~g})$ salmon. Various KEGG pathways involved in lipid, carbohydrate and protein metabolism were significantly $(P<0.05)$ regulated after feeding plant oil (PO) compared with fish oil. Statistical test was applied using hypergeometric test based on the number of PO-induced differential expressed genes $v$. total genes annotated to each KEGG ontology term. 


\section{Regulatory divergence of Ss4R gene duplicates}

Out of 8147 identified Ss4R duplicate pairs in total, about 5500 pairs had similar expression (log2 fold change between -0.5 and 0.5 ) in any tissue of salmon fed PO compared with FO. The rest of the pairs contained at least one DEG (log2 fold change $>0.5$ or $<-0.5)$ after feeding PO, including approximately 400 duplicate pairs with both increased or decreased expression ( $\log 2$ fold change both $>0.05$ or $<-0.05$ ), and around 100 pairs were reversely expressed. More interestingly, the sum and difference of fold change in expression of the two duplicate genes were larger in fingerlings than in juveniles (Fig. 2). This difference of regulation between fingerlings and juveniles was more distinct in intestinal tissues rather than in the liver. The total number of correlated and reversely expressed Ss4R duplicate pairs induced by PO was also reduced in intestinal tissues, whereas it increased in liver from fingerling to juvenile salmon (online Supplementary Table S4).

\section{Expressional differences of lipid metabolism genes} among tissues

Most of the 344 genes for lipid metabolism were highly expressed in pyloric caeca or liver, whereas few genes were highest expressed in stomach or hindgut (Fig. 3). In pyloric caeca, most lipid metabolism genes showed increasing expression from fingerling to juvenile stages, whereas very few genes had decreasing expression. Genes with increasing expression during development were found in hindgut and liver, but this pattern of change was mostly found in cholesterol and fatty acid metabolism pathways. Regardless of fish size, key genes in pathways of LC-PUFA elongation and desaturation, cholesterol biosynthesis and transport and lipoprotein formation were highly expressed only in pyloric caeca or liver (online Supplementary Table S5). These genes were also expressed in the hindgut, but at a lower level. The expression of these pathways was very low in the stomach. However, some genes encoding for fatty acid binding proteins were highly expressed in stomach and hindgut. Genes for fatty acid elongase elovl1b_1 and elovl $7 b a$ were also highly expressed in stomach, whereas other elongase and desaturase genes had very low expression levels.

\section{Regulation in lipid metabolism pathways in different tissues}

A total of seventy-six DEG were identified in pyloric caeca of fingerlings, whereas only eleven DEG were found in juveniles (online Supplementary Table S6). The DEG in fingerlings included twenty-six DEG covering each enzymatic step of the pathway for cholesterol biosynthesis (Fig. 4(a)). The expression of these genes was higher in fingerlings fed PO compared with those fed FO, indicating up-regulated cholesterol biosynthesis. However, these PO-induced differences of expression were evened out in juveniles and only five DEG were found. This was because of increased expression of genes for cholesterol biosynthesis in FO-fed juveniles compared with fingerlings, whereas the gene expression in PO-fed salmon was relatively unchanged between the two developmental stages.

On the other hand, the pathway of fatty acid elongation and desaturation was down-regulated in pyloric caeca after feeding

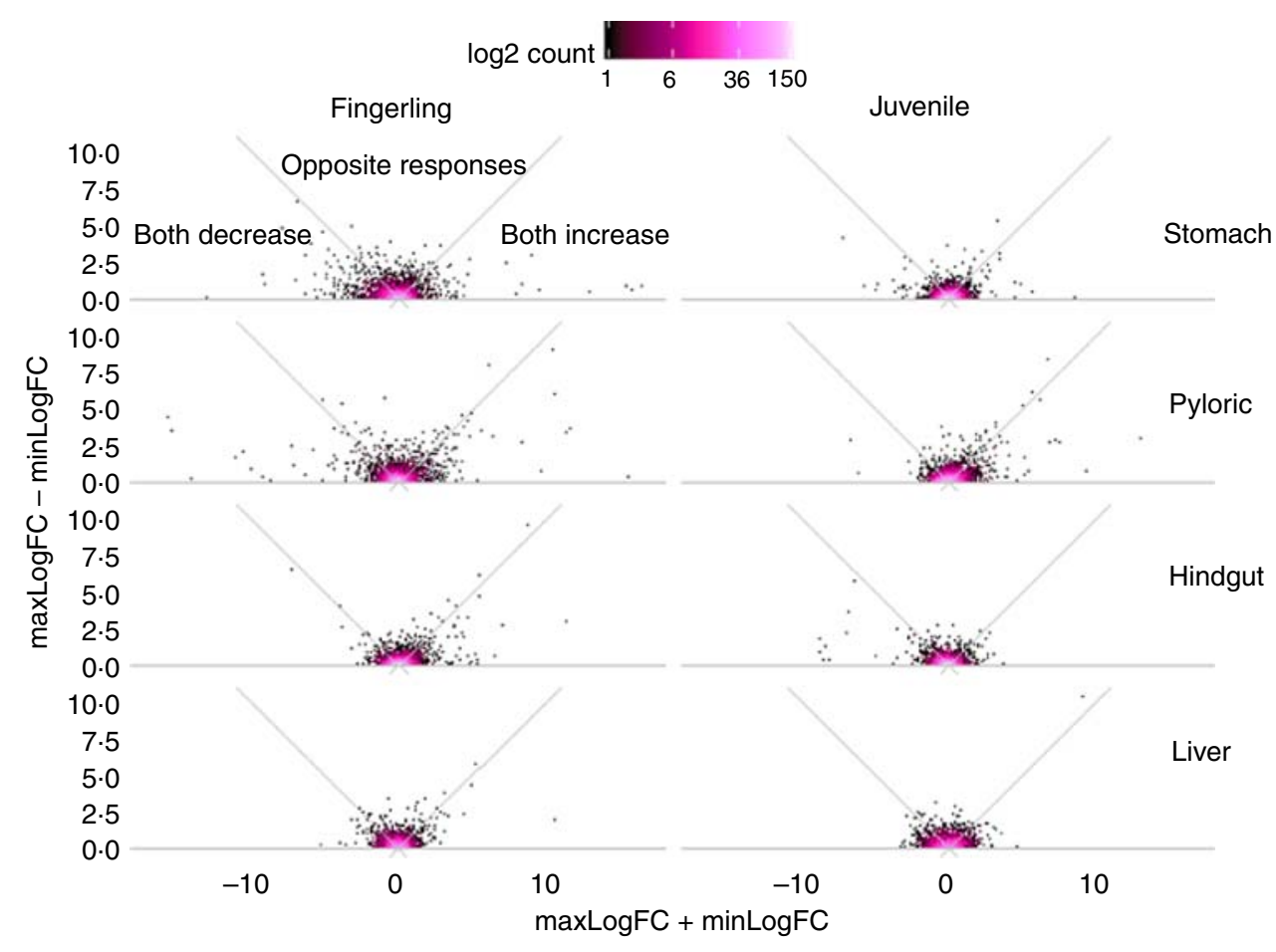

Fig. 2. Plant oil (PO) induced expressional change of salmonid-specific (Ss4R) duplicate genes in fingerling ( $2.5 \mathrm{~g})$ and juvenile (10 g) salmon. Each duplicate pair was separated into the gene with higher fold change $(\operatorname{maxLogFC})$ and lower fold change (minLogFC) after feeding PO, where maxLogFC $>$ minLogFC. The regulation divergence of Ss4R duplicate pairs can be separated into three groups: both increased (maxLogFC $>0, \min L o g F C>0$ ), both decreased (maxLogFC $<0$, $\operatorname{minLogFC}<0$ ) and opposite response. $\square$, Accumulation of duplicate pairs within each dot area. 
Fatty acid metabolism

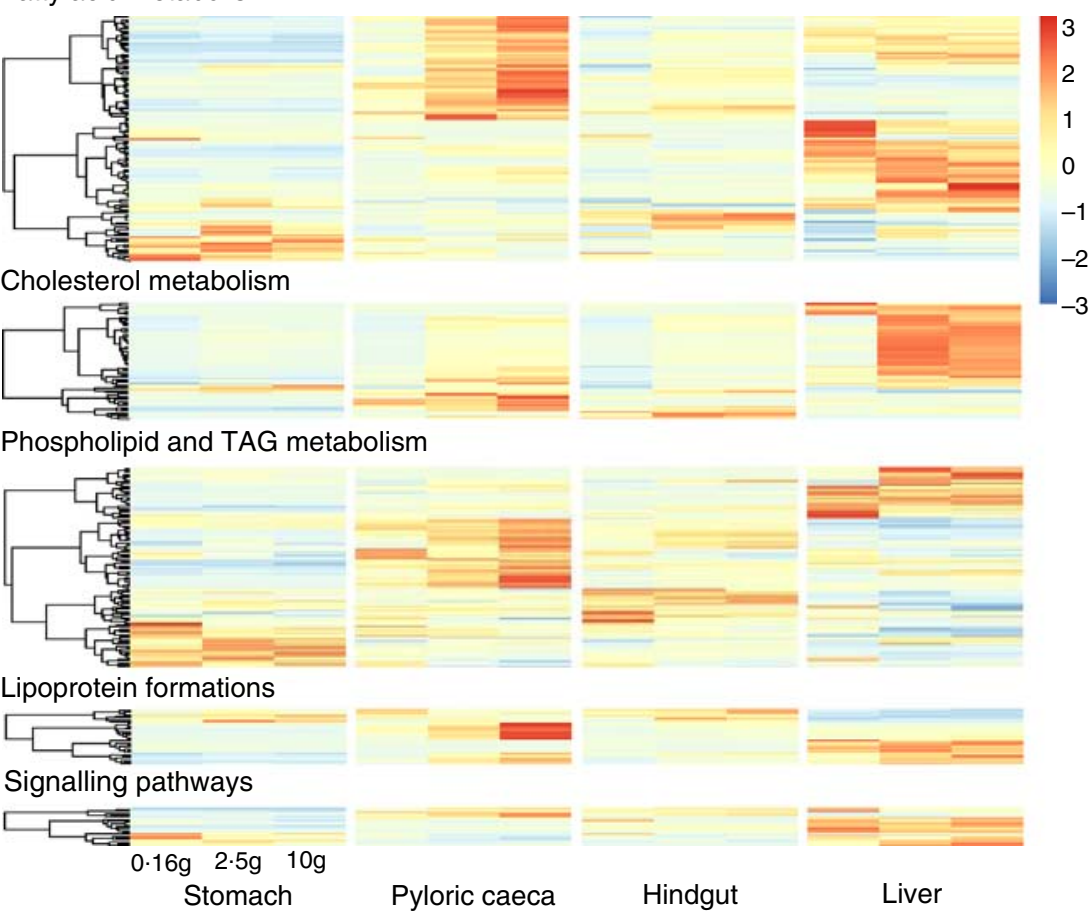

Fig. 3. Heatmap of 344 genes for lipid metabolism between different tissues. Different clusters of genes were dominant in each tissue and developmental stage of salmon. For each tissue, the three columns represent average transcripts per million (TPM) for samples of $0.16,2.5$ and $10 \mathrm{~g}$ salmon from left to right. The colour intensity is relative to the standard deviation from mean of TPM over developmental stages and tissues (row-scaled).

PO compared with FO (Fig. 4(b)). Key elongase gene elovl5_1 was strongly down-regulated ( $\log 2$ fold change $=-4 \cdot 3$, $q=7.6 \times 10^{-8}$ ) in fingerling salmon fed PO. Two desaturase genes $\Delta 5 \mathrm{fads}$ and $\Delta 6 \mathrm{fads} \mathbf{f}_{1}$, with $\Delta 5$ and $\Delta 6$ activities, respectively, were also highly expressed $(q<0.05)$ in FO-fed salmon compared with PO. Although no DEG was found in the pathway of elongation and desaturation in juvenile salmon, the expression of elovl5_1, $\Delta 5 \mathrm{fads}$ and $\Delta 6 \mathrm{fads} \_1$ was still higher in FO-compared with PO-fed salmon.

The expression of genes in phospholipid biosynthesis was mostly down-regulated in pyloric caeca of salmon fed PO (Fig. 4(c)). The $c h k b$ gene, which is involved in the first step of PtdCho biosynthesis ${ }^{(35)}$, was significantly $(q<0.05)$ higher expressed in salmon fingerlings fed FO compared with those fed PO. Genes in the subsequent two steps of PtdCho biosynthesis, pyct1bb_1 and chpt1_2, were also more highly expressed in FO than in PO. Although the expression of genes in PtdEtn biosynthesis was less clear than the gene in PtdCho pathways, slightly elevated expression of genes was found in FO-fed salmon (online Supplementary Table S6). Genes for sterol regulatory element-binding protein (SREBP) were regulated differently between duplicates (Fig. 4(d)). Genes srebp2a_1, srebp2a_2 and srebp $2 b$ were all significantly $(q<0.05)$ down-regulated in salmon fingerlings after feeding PO compared with FO, whereas the srebp1 genes srebp $1 b$ and srebp1d were up-regulated. The expressional differences between the two dietary groups were smaller in juvenile salmon compared with fingerlings, and no DEG was found.

Many genes involved in lipid absorption and transport were differentially expressed in pyloric caeca of fingerling salmon, whereas almost no DEG was found in juveniles (online Supplementary Table S6). The absorption of cholesterol was increased after feeding PO compared with FO, as two key genes scarb1 and npc1l1 were up-regulated in pyloric caeca of fingerlings (Fig. 5). Genes $a b c a 1 a b \_1$ and $a b c a 1 a b \_2$, which were involved in excretion of cholesterol into circulation ${ }^{(36)}$, were also highly expressed in PO-fed salmon. The absorption and intercellular transport of fatty acids in pyloric caeca were largely influenced by feeding PO. Most fatty acid transporter genes, namely $c d 36 \_2, f a b p 2 b, f a b p 6 \_1$ and fatp4_2, were more highly expressed in pyloric caeca of salmon fingerlings fed PO than FO, whereas fatp2c was more lowly expressed. The expression of $c d 36 \_2$ and fabp6_1 was still higher in salmon juvenile fed PO compared with FO. The pathway of lipoprotein formation in pyloric caeca was more lowly expressed in PO-fed salmon as key genes such as mtp_1, apoa1_1 and sar1ba_1 were down-regulated.

No clear size effect was found in liver on expressional differences of genes for lipid metabolism between fingerling and juvenile salmon (online Supplementary Table S6). The genes for cholesterol biosynthesis and fatty acid elongation and desaturation were all more highly expressed in salmon fed the PO diet compared with those fed the FO diet, although no DEG was identified. Two fatty acid transporter genes fabp6_1 and fabp3_1 and one cholesterol transporter gene npc1l1 were significantly $(q<0.05)$ more highly expressed in PO-fed salmon rather than FO (Fig. 5). This indicates up-regulated pathways of cholesterol biosynthesis and fatty acid elongation and desaturation in liver after feeding PO. Few DEG were found to be involved in the pathways of lipid metabolism in stomach and 
(a)

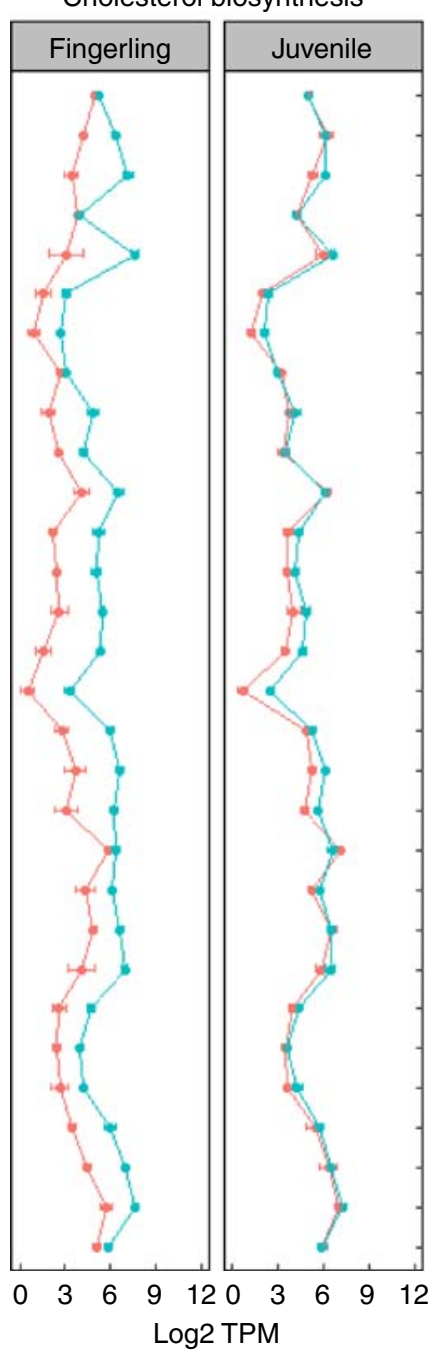

(b)

LC-PUFA elogation and desaturation

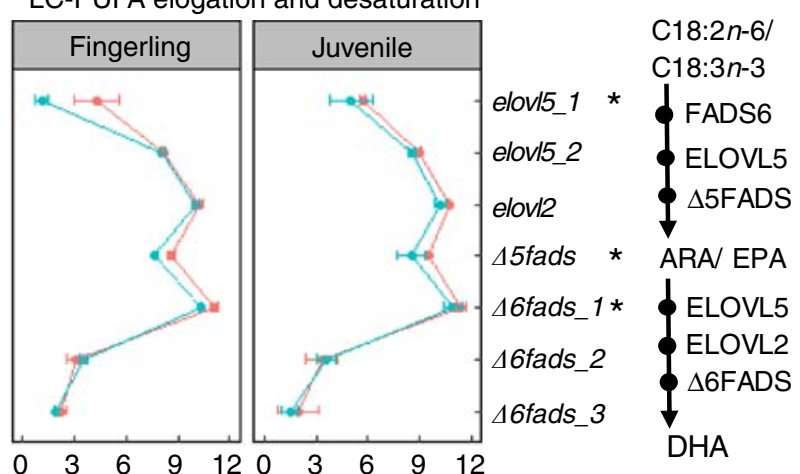

(c)

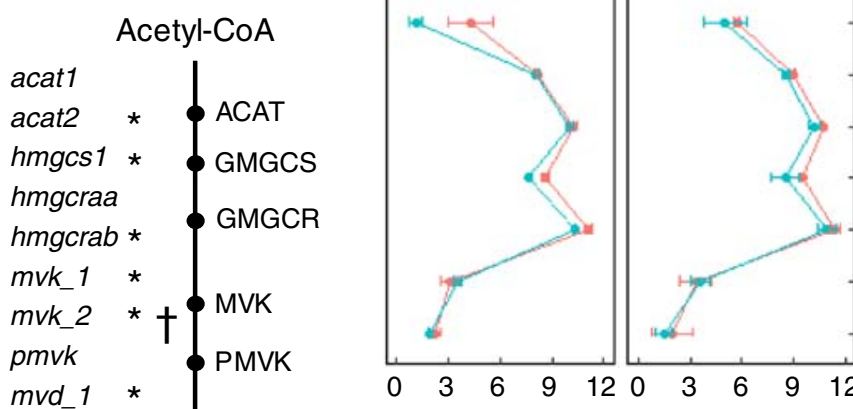

\section{PtdCho biosynthesis}

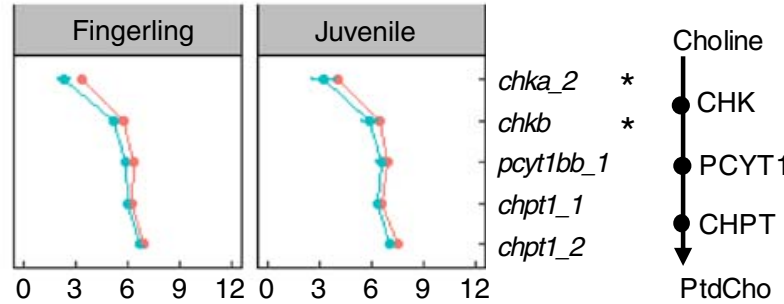

(d)

Srebp expression

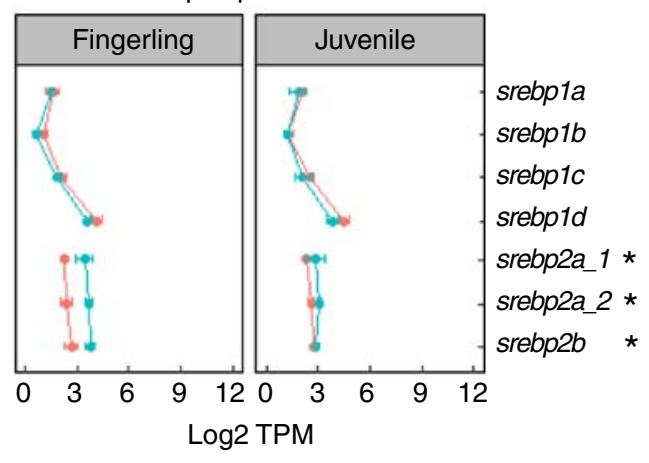

Fig. 4. Genes for lipid metabolism in pyloric caeca of salmon fingerlings $(2.5 \mathrm{~g})$ and juveniles $(10 \mathrm{~g})$ fed either fish oil (FO, --$)$ or plant oil $(\mathrm{PO},--)$. The expression of genes is compared in transcripts per million (TPM). * or $\dagger$, Differentially expressed gene $(q<0.05)$ in fingerling or juvenile salmon, respectively, fed PO compared with FO. (a) Expression of genes involved in each enzymatic step in the pathway of cholesterol biosynthesis. Gene duplicates are shown in a few steps of the pathway, which encode enzymes controlling the same chemical reaction. Higher expression of genes is shown for the PO group of fingerlings than for the FO group, whereas such differences are less clear in juvenile salmon. (b) Expression of seven key genes involved in long-chain PUFA (LC-PUFA) elongation and desaturation pathway. Expression was throughout slightly higher in salmon fed FO than PO diet. (c) Expression of five key genes involved in biosynthesis of phosphatidylcholine (PtdCho), which is the major phospholipid in the pyloric caeca of salmon. The expression of genes was higher in salmon fed FO diet than those fed PO diet. (d) Expression of seven srebp gene duplicates that play an important role in regulating genes in cholesterol and fatty acid biosynthesis in pyloric caeca of salmon. The srebp1 genes were more highly expressed in FO-fed salmon, whereas srebp2 genes were more highly expressed in PO-fed salmon.

hindgut of salmon after feeding PO (online Supplementary Table S6). Fatty acid binding protein genes fabp10a_1 and fabp10a_2 were significantly $(q<0.05)$ up-regulated in stomach after feeding PO, whereas fabp3_1 and $f a b p 2 b$ were upregulated in the hindgut.

\section{Discussion}

The present study aimed to increase current knowledge on how dietary PO affects lipid metabolism in developing salmon fingerlings $(2.5 \mathrm{~g})$ and juveniles $(10 \mathrm{~g})$. PO diets contained lower levels of LC-PUFA and cholesterol, which resulted in many biochemical and genetic feedback responses related to lipid metabolism in stomach, pyloric caeca, hindgut and liver. Many DEG were involved in cholesterol and fatty acid metabolism in pyloric caeca of fingerlings, with high fold changes of expression after feeding $\mathrm{PO}$ compared with FO. Both the number of DEG and their level of expression in PO- and FO-fed groups were much higher in fingerlings than in juveniles. This suggests that lipid metabolism regulation in fingerlings is more responsive to a plant-based diet than in juveniles. There were no DEG in liver related to lipid metabolism pathways in either fingerling or juvenile salmon after feeding PO. This contrasts a recent study on pre-smolt (approximately $50 \mathrm{~g}$ ) and post-smolt 


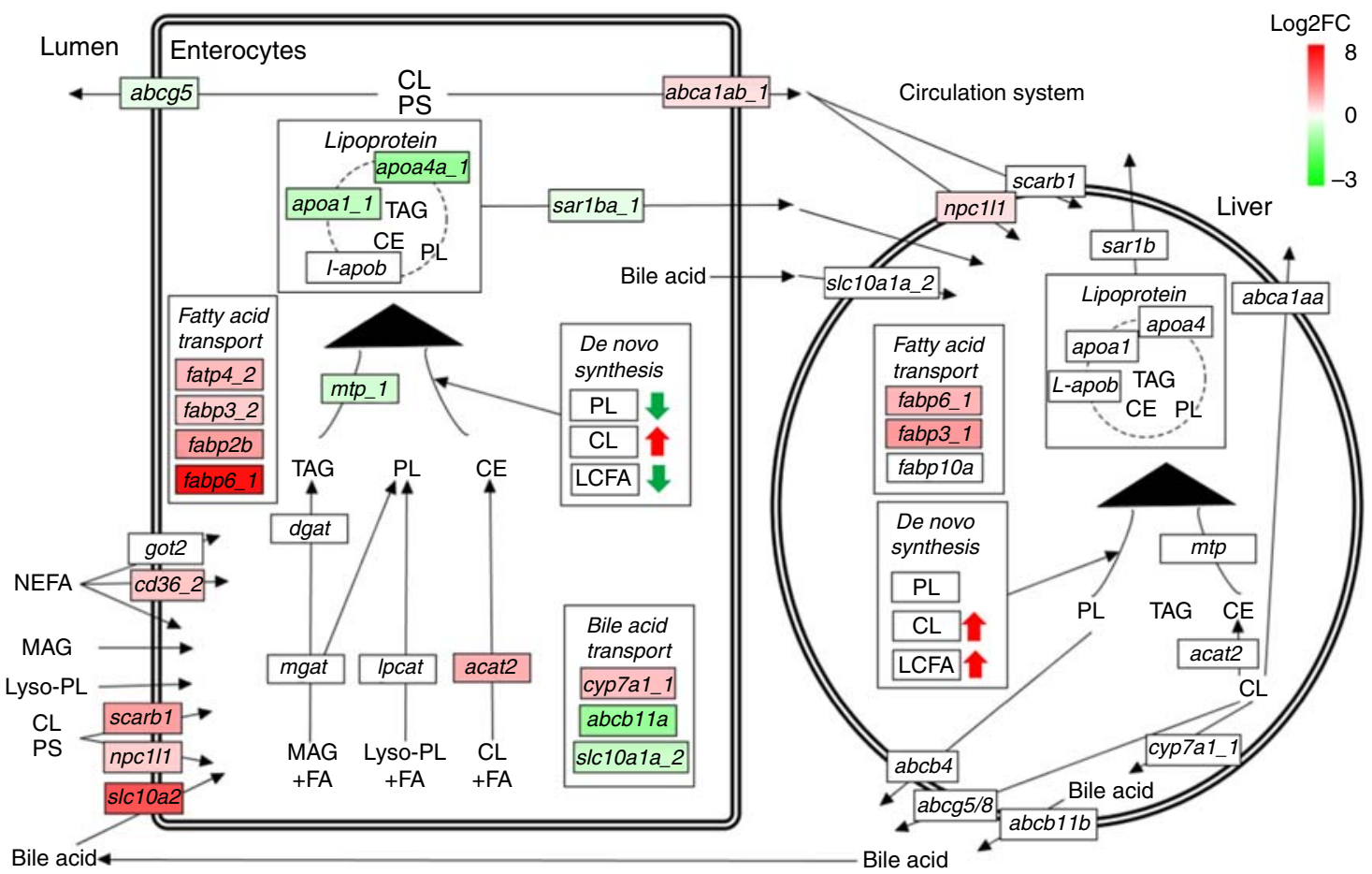

Fig. 5. Pathways of lipid metabolism in intestine and liver of salmon. Dietary NEFA, monoacylglycerol (MAG), lyso-phospholipids (lyso-PL), cholesterol (CL) and phytosterol (PS) are absorbed by intestinal enterocytes through either diffusion or a group of transport proteins. MAG, lyso-PL and CL are resynthesised into TAG, phospholipids (PL) and cholesteryl ester (CE), respectively, which are then packed into lipoprotein and effused to the circulatory system. Absorbed CL and PS can also be directly exported back to intestinal lumen or circulatory system through different transport proteins. Liver can take up lipoprotein residues and other nutrients from the circulatory system, and re-package them into new lipoproteins for circulation. Both intestine and liver have abilities of synthesising TAG, PL, CL and LCFA de novo. The labels indicate the highest expressed gene encoding the proteins. Only significantly expressed genes $(q<0.05)$ are marked in colour. The colour intensity is relative to the log2 fold change (Log2FC) of genes in plant oil-fed salmon compared with fish oil-fed salmon.

(approximately $200 \mathrm{~g}$ ) salmon fed the same PO and FO diets used in this present study, where a much higher number of lipid metabolism DEG were found in liver compared with intestine $^{(22)}$. Therefore, we suggest that fingerling and early juvenile salmon have an immature liver with low activity of lipid metabolism, which gradually increases as the fish develops.

De novo cholesterol synthesis was the most influenced pathway found in pyloric caeca of fingerlings when fed PO, with DEG covering every enzymatic step of the pathway. This is probably related to the reduced dietary cholesterol in fish fed $\mathrm{PO}$, inducing increased cholesterol de novo synthesis ${ }^{(37)}$. However, the requirement of cholesterol is probably lower in juveniles than in fingerlings, suggested by the up-regulation of cholesterol biosynthesis genes in fingerlings fed PO, while the expression of these genes remained similar in juveniles. A slight up-regulation of cholesterol biosynthesis was also found in liver, suggesting that liver was partly responsible for synthesising cholesterol to compensate for a dietary shortage. Besides de novo synthesis, fingerlings can also regulate their body cholesterol level through increasing the uptake of cholesterol while reducing its efflux. We found that the scarb1 and npc1l1 genes, which are responsible for cholesterol uptake ${ }^{(38)}$, and the $a b c a 1 a b \_1$ gene, which is responsible for transporting cholesterol into circulation ${ }^{(36)}$, were up-regulated in pyloric caeca of fingerlings after feeding $\mathrm{PO}^{(39)}$. On the other hand, the $a b c g 5$ gene, which is responsible for ejecting cholesterol back the intestinal lumen ${ }^{(40)}$, was down-regulated. The increased biosynthesis and absorption of cholesterol can be regulated by SREBP, which are transcription factors controlling cellular cholesterol content $^{(41)}$. Two groups of SREBP have been identified in mammals, from which SREPB-2 is more selective for genes involved in cholesterol homoeostasis ${ }^{(42,43)}$. The higher expression of srebp2 genes in pyloric caeca of PO-fed salmon was in line with the up-regulation of cholesterol biosynthesis genes, in agreement with a previous finding in seabream ${ }^{(44)}$.

Dietary PO often contains high amounts of phytosterols, which can compete with cholesterols for space in the micelles and reduce cholesterol absorption in the intestine ${ }^{(20,45)}$. Owing to its similarity to cholesterol, phytosterols can be taken up in the intestine and transported to fish body tissues by the same transport proteins used for cholesterol ${ }^{(20,39)}$. However, phytosterols do not appear to be esterified by acyl-CoA:cholesterol O-acyltransferase 2 (ATAC2) and packed into lipoproteins with TAG and phospholipids ${ }^{(46)}$. Rather, phytosterols seem to mainly be exported back into the intestinal lumen through ATP-binding cassette transporter sub-family G (ABCG5/8), whereas the rest can be transported into circulation through ATP-binding cassette transporter sub-family A (ABCA1) ${ }^{(39,40)}$. In fingerlings, the increase in expression of $a b c a 1 a b \_1$, encoding ABCA1, and the decrease in expression $a b c g 5$, encoding ABCG5, could increase the accumulation of phytosterols in the intestinal tissue, and consequently transport into circulation. Previous studies have shown that dietary phytosterols did not influence growth and performance of parr or adult salmon ${ }^{(23,47)}$. However, whether 
phytosterol has an impact on fingerling or juvenile salmon needs to be further investigated.

In general, the rate of changes in fatty acid composition after feeding PO compared with FO was consistently larger in fingerlings than in juveniles. This suggests that salmon fingerlings have less metabolic regulatory control when fed the PO diet than juveniles. Regarding the lipid classes, the fatty acid composition of TAG was often similar to the composition of dietary fatty acids, whereas phospholipids, which are the membrane lipids and thus play structural roles, are often more strictly regulated $^{(48)}$. However, feeding of PO had a major effect on most fatty acids in both PtdCho and PtdEtn. This suggests that PtdCho and PtdEtn can use a wide range of diacylglycerol with different fatty acid compositions for their carbon skeleton ${ }^{(17)}$. Dietary PO had particularly little effect on the percentage of PtdCho-DHA and almost no effect on PtdEtn-DHA, suggesting that DHA was selectively retained in phospholipid when salmon were fed $\mathrm{PO}^{(49)}$. Similar to previous findings in adult trout ${ }^{(50)}$, a higher percentage of $18: 1 n-9$ and ARA was more efficiently retained in stomach than in pyloric caeca and hindgut. The high percentage of ARA was probably used for eicosanoid synthesis and gastric mucosa protection ${ }^{(50)}$.

Although dietary PO increased the $18: 3 n-3$ and $18: 2 n-6$ contents and decreased ARA, EPA and DHA percentages in pyloric caeca of fingerlings, the expression of elongation (elovl5_1) and desaturation ( $\Delta 5 \mathrm{fads}$ and $\Delta 6 \mathrm{fads})$ genes was depressed. This was associated with down-regulation of srebp1 genes, which are the primary transcription factors for endogenous production of LC-PUFA in salmon ${ }^{(51)}$. These results are in disagreement with the generally accepted conclusion that dietary PO stimulates the expression of genes for elongation and desaturation in fish ${ }^{(15,52)}$. The reason for the downregulation of elongation and desaturation in pyloric caeca of PO-fed fingerlings remains unclear. A similar result has been found using primary cultures of enterocytes obtained from fish fed linseed oil ${ }^{(16)}$, which was also used in this study. The activity of elongation and desaturation in enterocytes was significantly reduced in PO compared with FO fed salmon, whereas in hepatocytes the activity was increased ${ }^{(16)}$. We also identified slight up-regulation of elovl5, elovl2, $\Delta 5 \mathrm{fads}$ and $\Delta$ ffads genes in liver following PO feeding. The reason for the reverse regulation of elongation and desaturation between pyloric caeca and liver was not clear.

For the first time in salmon, we identified a variety of genes involved in fatty acid transport pathways and measured their expression among intestinal and liver tissues. Pyloric caeca are the predominant regions for nutrient and fatty acid uptake in fish. This explained the higher expression of fatty acid transporter $c d 36$ and fatp genes in the pyloric caeca than in other intestinal tissues. These genes had lower, but considerable, expression in hindgut, in line with a lower but still notable fatty acid uptake in salmonids ${ }^{(4,17)}$. Unlike mammalian CD36 mRNA expressions ${ }^{(53,54)}$, salmon $c d 36 \_1$ and $c d 36 \_2$ genes had low expression levels in liver. However, we found that fatp genes had higher expression in liver than intestinal tissues, suggesting that it is primarily responsible for fatty acid uptake in the tissue. Dietary PO stimulated varieties of fatp and $c d 36$ genes in pyloric caeca tissues, indicating increased activity of fatty acid uptake and transport. On the other hand, fatp $2 c$ was downregulated in pyloric caeca, probably owing to the different fatty acid preference by fatty acid transporters. A previous study has demonstrated that dietary rapeseed oil with high $18: 1 n-9$ could reduce $c d 36$ and fatp expression in salmon ${ }^{(55)}$. Furthermore, the intracellular transport of fatty acids in liver and intestine can be largely affected by dietary PO, as different fabp genes were differentially expressed in stomach, pyloric caeca, hindgut and liver.

Bile acids are synthesised from cholesterol by cholesterol $7 \alpha-$ hydroxylase (CYP7A1) in liver and transported through transporter ATP-binding cassette, sub-family B member 11 (ABCB11) into the gallbladder where they are mixed with phospholipids, then transported through ABCB4 to form mature bile. In the intestine, bile acids can be reabsorbed through the SLC10A2 protein and returned to portal circulation before being taken up by liver through SCL10A1 protein and repumped into the gallbladder $^{(56)}$. In mammals, the cyp7a1, abcb11, abcb4 and scl10a1 genes are highly expressed in liver (available on https://www.proteinatlas.org/). We also found high expression levels of cyp 7a1, abcb11, abcb4 and scl10a1 genes in pyloric caeca being differentially expressed in fingerlings fed PO compared with those fed FO. This could suggest an additional bile acid production and transport in pyloric caeca, which could be especially important for salmon at early developmental stages. However, the function and activity of the proteins encoded by these genes are largely unknown in salmon, and it remains unclear whether these proteins have similar functions as in liver.

Whole-genome duplication is believed to play a major role in evolution, providing extra copies of genes with sub- or neofunctionalisation, on which selection can take place ${ }^{(57)}$. This study showed that 23\% (350 out of 1512) of the salmon duplicated pairs were reversely regulated after feeding PO compared with FO, indicating a high regulatory divergence of duplicates in salmon. The incomplete rediploidisation of the salmon genome has increased the complexity of the pathways of lipid metabolism in the species. It has been suggested that salmon duplicates may have led to diverged gene expressions between different tissues such as intestine and liver ${ }^{(32)}$. However, as the intestine consists of different regions with distinct functions, it is physiologically and genetically important to analyse the functional divergence of these regions separately. The regulation of salmon duplicates was different between stomach, pyloric caeca and hindgut. This is in accordance with the physiological similarity between the tissues, where pyloric caeca is responsible for digestion and absorption, whereas stomach is more responsible for pepsin and acid degradation and physical decomposition of the feed.

In conclusion, the present study has provided a systemic overview of PO-induced changes in lipid metabolism in intestine and liver of salmon during early stages of development. By comparing the expression levels of lipid metabolism genes between $\mathrm{PO}$ and FO, we found that salmon has a more sensitive response to $\mathrm{PO}$ at fingerling stages compared with juvenile. In particular, the expression levels of genes in cholesterol absorption and biosynthesis and transport pathways in pyloric caeca were highly up-regulated in fingerlings, suggesting a higher requirement of dietary cholesterol. An increased 
cholesterol biosynthesis pathway was also found in liver, although no clear difference was found between fingerling and juvenile stages. The fatty acid elongation and desaturation pathways were down-regulated in pyloric caeca of fingerlings after feeding PO diet, but up-regulated in liver. However, further in vivo or in vitro studies are required to confirm the present findings on protein level. We also suggest future studies on dietary requirement of lipids in fingerling stages of salmon, as the fish seemed to have insufficient capacity of lipid metabolism at early stages of development.

\section{Acknowledgements}

The authors thank Hanne Hellerud Hansen, Thomas Nelson Harvey and the Centre for Integrative Genetics for RNA-Seq sample preparation and data analysis. The authors thank Dr Maren Mommens and AquaGen AS for providing experimental facilities and other practical information, and Dr Keshuai Li for support on lipid analysis.

The design, analysis and writing of this article was supported by no specific grant from Department of Biology, Norwegian University of Science and Technology (NTNU). The RNA-Seq and data analysis were financed by the Research Council of Norway (GenoSysFat, grant no. 244164), (DigiSal, grant no. 248792). The authors thank AquaGen AS for providing the fish and for contribution of running the experiment. The feeds were produced by Dominic Nanton and Cargill Innovation Centre Dirdal. The authors also thank the China Scholarship Council for providing financial support to Yang Jin for his $\mathrm{PhD}$ study.

Y. J., Y. O. and R. E. O. designed the research; Y. J. and G. B. G. performed the RNAseq and transcriptomic data analysis; Y. J. performed the lipid analysis; J. O. V. and S. R. S. guided the transcriptomic analysis and revised the manuscript. Y. O. and R. E. O. guided the lipid analysis and revised the manuscript. M. $\varnothing$., N. S. and S. A. K. provided input on the experimental design, carried out the feeding trial and sampling and reviewed the manuscript. All authors read and approved the final manuscript.

The authors declare that there are no conflicts of interest.

\section{Supplementary material}

For supplementary material/s referred to in this article, please visit https://doi.org/10.1017/S0007114518001885

\section{References}

1. Food and Agriculture Organization (2014) The State of World Fisheries and Aquaculture 2014. Rome: FAO.

2. Ytrestoyl T, Aas TS \& Asgard T (2015) Utilisation of feed resources in production of Atlantic salmon (Salmo salar) in Norway. Aquaculture 448, 365-374.

3. Carmona-Antonanzas G, Taylor JF, Martinez-Rubio L, et al. (2015) Molecular mechanism of dietary phospholipid requirement of Atlantic salmon, Salmo solar, fry. Biochim Biophys Acta 1851, 1428-1441.

4. Olsen RE, Myklebust R, Kaino T, et al. (1999) Lipid digestibility and ultrastructural changes in the enterocytes of Arctic char
(Salvelinus alpinus L.) fed linseed oil and soybean lecithin. Fish Physiol Biochem 21, 35-44.

5. Olsen RE, Myklebust R, Ringo E, et al. (2000) The influences of dietary linseed oil and saturated fatty acids on caecal enterocytes in Arctic char (Salvelinus alpinus L.): a quantitative ultrastructural study. Fish Physiol Biochem 22, 207-216.

6. Tocher DR, Bell JG, McGhee F, et al. (2003) Effects of dietary lipid level and vegetable oil on fatty acid metabolism in Atlantic salmon (Salmo salar L.) over the whole production cycle. Fish Physiol Biochem 29, 193-209.

7. Calzada A, Medina A \& Canales MLG (1998) Fine structure of the intestine development in cultured sea bream larvae. J Fish Biol 53, 340-365.

8. Jin Y, Olsen RE, Østensen M-A, et al. (2018) Transcriptional development of phospholipid and lipoprotein metabolism in different intestinal regions of Atlantic salmon (Salmo salar) fry. BMC Genomics 19, 253.

9. Saunders DR (1991) Absorption of short-chain fatty acids in human stomach and rectum. Nutr Res 11, 841-847.

10. Lazo JP, Darias MJ \& Gisbert E (2011) Ontogeny of the digestive tract. In Larval Fish Nutrition, pp. 3-46 [GJ Holt, editor]. Chichester: Wiley-Blackwell.

11. Sahlmann C, Gu J, Kortner TM, et al. (2015) Ontogeny of the digestive system of Atlantic salmon (Salmo salar L.) and effects of soybean meal from start-feeding. PLOS ONE 10, e0124179.

12. Bligh EG \& Dyer WJ (1959) A rapid method of total lipid extraction and purification. Can J Biochem Phys 37, 911-917.

13. Rennie S, Huntingford FA, Loeland AL, et al. (2005) Long term partial replacement of dietary fish oil with rapeseed oil; effects on egg quality of Atlantic salmon Salmo salar. Aquaculture 248, 135-146.

14. Sargent JR, Henderson RJ \& Tocher DR (1989) The lipids. In Fish Nutrition, vol. 2, pp. 153-218 [JE Halver, editor]. New York: Academic Press.

15. Tocher DR (2015) Omega-3 long-chain polyunsaturated fatty acids and aquaculture in perspective. Aquaculture 449, 94-107.

16. Tocher DR, Fonseca-Madrigal J, Bell JG, et al. (2002) Effects of diets containing linseed oil on fatty acid desaturation and oxidation in hepatocytes and intestinal enterocytes in Atlantic salmon (Salmo salar). Fish Physiol Biochem 26, 157-170.

17. Olsen RE, Dragnes BT, Myklebust R, et al. (2003) Effect of soybean oil and soybean lecithin on intestinal lipid composition and lipid droplet accumulation of rainbow trout, Oncorhynchus mykiss Walbaum. Fish Physiol Biochem 29, 181-192.

18. Leaver MJ, Villeneuve LAN, Obach A, et al. (2008) Functional genomics reveals increases in cholesterol biosynthetic genes and highly unsaturated fatty acid biosynthesis after dietary substitution of fish oil with vegetable oils in Atlantic salmon (Salmo salar). BMC Genomics 9, 299.

19. Van der Wulp MYM, Verkade HJ \& Groen AK (2013) Regulation of cholesterol homeostasis. Mol Cell Endocrinol 368, 1-16.

20. Liland NS, Espe M, Rosenlund G, et al. (2013) High levels of dietary phytosterols affect lipid metabolism and increase liver and plasma TAG in Atlantic salmon (Salmo salar L.). Br J Nutr 110, 1958-1967.

21. Szterk A, Roszko M, Sosińska E, et al. (2010) Chemical composition and oxidative stability of selected plant oils. J Am Oil Chem Soc 87, 637-645.

22. Gillard G, Harvey TN, Gjuvsland A, et al. (2018) Life-stage associated remodeling of lipid metabolism regulation in Atlantic salmon. Mol Ecol 27, 1200-1213.

23. Sissener NH, Liland NS, Holen E, et al. (2017) Phytosterols are not involved in the development of fatty liver in plant oil fed Atlantic salmon (Salmo salar) at high or low water temperature. Aquaculture 480, 123-134. 
24. Monroig O, Navarro JC \& Tocher DR (2011) Long-chain polyunsaturated fatty acids in fish: recent advances on desaturases and elongases involved in their biosynthesis. Proceedings of the XI International Symposium on Aquaculture Nutrition, pp. 257-282.

25. Tocher DR (2010) Fatty acid requirements in ontogeny of marine and freshwater fish. Aquac Res 41, 717-732.

26. Ruyter B, Rosjo C, Einen O, et al. (2000) Essential fatty acids in Atlantic salmon: effects of increasing dietary doses of $n-6$ and $n-3$ fatty acids on growth, survival and fatty acid composition of liver, blood and carcass. Aquacult Nutr 6, 119-127.

27. Bou M, Berge GM, Baeverfjord G, et al. (2017) Requirements of $n-3$ very long-chain PUFA in Atlantic salmon (Salmo salar L.): effects of different dietary levels of EPA and DHA on fish performance and tissue composition and integrity. $\mathrm{Br} J$ Nutr 117, 30-47.

28. Tocher DR (2003) Metabolism and functions of lipids and fatty acids in teleost fish. Rev Fish Sci 11, 107-184.

29. Sargent J, Bell G, McEvoy L, et al. (1999) Recent developments in the essential fatty acid nutrition of fish. Aquaculture 177, 191-199.

30. Macqueen DJ \& Johnston IA (2014) A well-constrained estimate for the timing of the salmonid whole genome duplication reveals major decoupling from species diversification. Proc Biol Sci 281, 20132881.

31. Wright JE Jr, Johnson K, Hollister A, et al. (1983) Meiotic models to explain classical linkage, pseudolinkage, and chromosome pairing in tetraploid derivative salmonid genomes. Isozymes Curr Top Biol Med Res 10, 239-260.

32. Lien S, Koop BF, Sandve SR, et al. (2016) The Atlantic salmon genome provides insights into rediploidization. Nature $\mathbf{5 3 3}$, 200-205.

33. Folch J, Lees M \& Sloane Stanley GH (1957) A simple method for the isolation and purification of total lipides from animal tissues. J Biol Chem 226, 497-509.

34. Olsen RE \& Henderson RJ (1989) The rapid analysis of neutral and polar marine lipids using double-development HPTLC and scanning densitometry. J Exp Mar Biol Ecol 129, 189-197.

35. Vance JE \& Vance DE (2004) Phospholipid biosynthesis in mammalian cells. Biochem Cell Biol 82, 113-128.

36. Oram JF \& Vaughan AM (2000) ABCA1-mediated transport of cellular cholesterol and phospholipids to HDL apolipoproteins. Curr Opin Lipidol 11, 253-260.

37. Siperstein MD (1970) Regulation of cholesterol biosynthesis in normal and malignant tissues. Curr Top Cell Regul 2, 65-100.

38. Jia L, Betters JL \& Yu LQ (2011) Niemann-Pick C1-Like 1 (NPC1L1) protein in intestinal and hepatic cholesterol transport. Annu Rev Physiol 73, 239-259.

39. Fumeron F, Bard JM \& Lecerf JM (2017) Interindividual variability in the cholesterol-lowering effect of supplementation with plant sterols or stanols. Nutr Rev 75, 134-145.

40. Field FJ, Born E \& Mathur SN (2004) LXR/RXR ligand activation enhances basolateral efflux of beta-sitosterol in CaCo-2 cells. J Lipid Res 45, 905-913.

41. Brown MS \& Goldstein JL (1999) A proteolytic pathway that controls the cholesterol content of membranes, cells, and blood. Proc Natl Acad Sci U S A 96, 11041-11048.

42. Horton JD, Shimomura I, Brown MS, et al. (1998) Activation of cholesterol synthesis in preference to fatty acid synthesis in liver and adipose tissue of transgenic mice overproducing sterol regulatory element-binding protein-2. J Clin Invest 101, 2331-2339.

43. Pai JT, Guryev O, Brown MS, et al. (1998) Differential stimulation of cholesterol and unsaturated fatty acid biosynthesis in cells expressing individual nuclear sterol regulatory elementbinding proteins. J Biol Chem 273, 26138-26148.

44. Houston SJS, Karalazos V, Tinsley J, et al. (2017) The compositional and metabolic responses of gilthead seabream (Sparus aurata) to a gradient of dietary fish oil and associated n-3 long-chain PUFA content. Br J Nutr 118, 1010-1022.

45. Amemiya-Kudo M, Shimano H, Hasty AH, et al. (2002) Transcriptional activities of nuclear SREBP-1a, $-1 \mathrm{c}$, and -2 to different target promoters of lipogenic and cholesterogenic genes. J Lipid Res 43, 1220-1235.

46. Temel RE, Gebre AK, Parks JS, et al. (2003) Compared with acyl-CoA:cholesterol $O$-acyltransferase (ACAT) 1 and lecithin: cholesterol acyltransferase, ACAT2 displays the greatest capacity to differentiate cholesterol from sitosterol. J Biol Chem 278, 47594-47601.

47. Gu M, Kortner TM, Penn M, et al. (2014) Effects of dietary plant meal and soya-saponin supplementation on intestinal and hepatic lipid droplet accumulation and lipoprotein and sterol metabolism in Atlantic salmon (Salmo salar L.). $\mathrm{Br} \mathrm{J}$ Nutr 111, 432-444.

48. Sargent JR, Tocher DR \& Bell JG (2002) The lipids. Fish Nutr 3, 181-257.

49. Stubhaug I, Lie $\varnothing$ \& Torstensen BE (2007) Fatty acid productive value and $\beta$-oxidation capacity in Atlantic salmon (Salmo salar L.) fed on different lipid sources along the whole growth period. Aquacult Nutr 13, 145-155.

50. Tarnawski A, Hollander D, Stachura J, et al. (1989) Protection of the rat gastric mucosa against aspirin injury by arachidonic acid: a dietary prostaglandin precursor fatty acid. Eur J Clin Invest 19, 278-290.

51. Carmona-Antonanzas G, Tocher DR, Martinez-Rubio L, et al. (2014) Conservation of lipid metabolic gene transcriptional regulatory networks in fish and mammals. Gene 534, 1-9.

52. Kjaer MA, Ruyter B, Berge GM, et al. (2016) Regulation of the omega-3 fatty acid biosynthetic pathway in Atlantic salmon hepatocytes. PLOS ONE 11, e0168230.

53. Chen M, Yang Y, Braunstein E, et al. (2001) Gut expression and regulation of FAT/CD36: possible role in fatty acid transport in rat enterocytes. Am J Physiol Endocrinol Metab 281, E916-E923.

54. Wilson CG, Tran JL, Erion DM, et al. (2016) Hepatocytespecific disruption of $\mathrm{cd} 36$ attenuates fatty liver and improves insulin sensitivity in HFD-fed mice. Endocrinology 157, $570-585$.

55. Torstensen BE, Nanton DA, Olsvik PA, et al. (2009) Gene expression of fatty acid-binding proteins, fatty acid transport proteins ( $\mathrm{cd} 36$ and FATP) and $\beta$-oxidation-related genes in Atlantic salmon (Salmo salar L.) fed fish oil or vegetable oil. Aquacult Nutr 15, 440-451.

56. Trauner M \& Boyer JL (2003) Bile salt transporters: molecular characterization, function, and regulation. Physiol Rev 83, 633-671.

57. Wolfe KH (2001) Yesterday's polyploids and the mystery of diploidization. Nat Rev Genet 2, 333-341. 